\title{
Technology research on Public welfare map adaptively and quickly producting
}

\author{
Zhu Xiuli a, *, Zhao Yong a , Liu Wanzeng a , Wu Chenchen ${ }^{a}$,Wang Zhongxiang a \\ ${ }^{a}$ National Geomatics Center of China; No. 28 Lianhuachi West Road, Haidian District, Beijing100830, China; \\ e-mail:zhuxiuli@ngcc.cn,
}

Keywords: Public welfare maps, quickly producting technology of map, map projection, adaptively producting map

\begin{abstract}
:
Public welfare maps are thematic maps for macro-planning, emergency relief, decision-making and daily office work of government departments. Public welfare maps are used to serve the government.

Public welfare maps are used to serve the government In recent years, the state has become more and more demanding for the rapid guarantee service work of public welfare maps of surveying and mapping product. It shows that the demand for high-potential, high-quality geographic information high-efficiency and spiritual services is becoming more and more urgent. Therefore, research The rapid production technology of public welfare maps has also become an urgent requirement. In response to this demand, this paper studies the key issues of rapid map production, such as rapid positioning, projection and scale adaptation, layout and off-line adaptive self-adaptation, and its solutions.

Firstly, this paper introduces the necessity of adaptive mapping, the model and algorithm of adaptive mapping. Then introduces projection and scale adaptation, layout and out-of-map integer adaptation. Finally, based on the above research results, a rapid producting system for public welfare maps is developed. We compile sample map and test performance using the system. The result show that the paper's methods can improve the efficiency of Public welfare map producting, and provide powerful technical support for quickly service.
\end{abstract}

